\title{
Quality Changes in Fundus Images of Pseudophakic Eyes
}

\section{Cambios en la calidad de la imagen retiniana de los ojos pseudofáquicos}

\author{
María S. Millán 1,5*, Andrés G. Marrugo ${ }^{2 *}$, Francisco Alba-Bueno 1,3,S \\ 1. Grupo de Óptica Aplicada y Procesado de Imagen, Facultat d'Òptica i Optometria de Terrassa, \\ Universitat Politècnica de Catalunya (UPC), BARCELONATECH, Terrassa (Barcelona), España \\ 2. Facultad de Ingeniería, Universidad Tecnológica de Bolívar, Cartagena, Colombia \\ 3. Department of Medical \& Regulatory Affairs of Alcon-Novartis \\ (*)E-mail: m.millan@upc.edu ,agmarrugo@utbvirtual.edu.co \\ $\mathrm{S}:$ SEDOPTICA member
}

Received: $13 / 02 / 2018 \quad$ Accepted: 05/10/2018

DOI: 10.7149/OPA.51.4.50015

\begin{abstract}
:
We consider the changes produced in the quality of eye fundus images of patients before and after cataract surgery with intraocular lens implantation and propose a measure to quantitatively assess those changes. Several factors concerning the optical system of the eye, the ocular media, and the specific characteristics of the implanted intraocular lens, may influence the quality of the digital image acquired with a non-mydriatic retinal camera. We illustrate our study with several cases taken from the clinical practice. A pair of pre- and post-operative eye fundus images represents each case. To measure image quality, we carry out a calculation of image anisotropy on each image. The results show that after surgery with intraocular lens implant, a fundus image is usually much brighter, sharp and higher quality. However, this rule of thumb can be altered by several conditions. For example, the multifocal design of the intraocular implant after a clear lens extraction. The anisotropy-based measure has revealed to be a suitable tool to quantitatively assess quality changes between pre-and post-operative fundus images.
\end{abstract}

Key words: Digital image analysis; image quality, eye fundus image; intraocular lens, pseudophakia, non-mydriatic retinal camera

\section{RESUMEN:}

En este trabajo estudiamos los cambios producidos en la calidad de imágenes del fondo de ojo de pacientes antes y después de cirugía de catarata con implantación de lente intraocular, y proponemos una medida para evaluar esos cambios cuantitativamente. Diversos factores relacionados con el sistema óptico del ojo, los medios oculares y las características específicas de la lente intraocular implantada pueden influir en la calidad de la imagen digital adquirida con una cámara de retina no midriática. Ilustramos el estudio con varios casos tomados de la práctica clínica. Un par de imágenes del fondo de ojo pre y post-operatorias representa cada caso. Para medir la calidad de la imagen, llevamos a cabo un cálculo de la anisotropía en cada imagen. Los resultados muestran que después de la cirugía con implante de lente intraocular, una imagen de fondo de ojo suele ser mucho más brillante, nítida y de mayor calidad. Sin embargo, esta regla práctica puede verse alterada por varias condiciones. Por ejemplo, el diseño multifocal del implante intraocular después de una extracción de cristalino transparente. La medida basada en la anisotropía demostró ser una herramienta adecuada para evaluar cuantitativamente los cambios de calidad entre las imágenes del fondo de ojo pre y postoperatorias.

Palabras clave: Análisis digital de imagen, calidad de imagen, imagen de fondo de ojo, lente intraocular, pseudofaquia, cámara no-midriática de retina 


\section{REFERENCES AND LINKS / REFERENCIAS Y ENLACES}

[1] M. D. Abramoff, M. Garvin, and M. Sonka, "Retinal Imaging and Image Analysis," Biomedical Engineering, IEEE Reviews in, 3, pp. 169-208, (2010).

[2] L. Abdel-Hamid, A. El-Rafei, S. El-Ramly, G. Michelson, and J. Hornegger, "Retinal image quality assessment based on image clarity and content," J. Biomed. Opt., 21(9), p. 096007, (2016).

[3] A. D. Fleming, S. Philip, K. A. Goatman, J. A. Olson, and P. F. Sharp, "Automated assessment of diabetic retinal image quality based on clarity and field definition," Invest Ophth Vis Sci, 47(3), pp. 1120-1125, (2006).

[4] A. G. Marrugo, M. S. Millan, G. Cristóbal, S. Gabarda, and H. C. Abril, "No-reference quality metrics for eye fundus imaging," presented at the CAIP'11: Proceedings of the 14th international conference on Computer analysis of images and patterns, 6854 pp. 486-493 (2011).

[5] L. Giancardo, M. D. Abramoff, E. Chaum, T. Karnowski, F. Meriaudeau, and K. Tobin, "Elliptical local vessel density: A fast and robust quality metric for retinal images.," Annual Int Conf of the IEEE Engineering in Medicine and Biology Society, 1(4), pp. 3534-1179, (2008).

[6] J. L. Alió, P. Schimchak, R. Montés-Micó, and A. Galal, "Retinal image quality after microincision intraocular lens implantation," Journal of Cataract \& Refractive Surgery, 31(8), pp. 1557-1560, (2005).

[7] D. Siedlecki, M. Zając, and J. Nowak, "Retinal images in a model of a pseudophakic eye with classic and hybrid intraocular lenses," Journal of Modern Optics, 55(4) pp. 653-669, (2008).

[8] H. Guo, A. V. Goncharov, and C. Dainty, "Comparison of retinal image quality with spherical and customized aspheric intraocular lenses," Biomedical Optics Express, 3(4), pp. 681-691, (2012).

[9] H. Zhao and M. A. Mainster, "Ghost Images And Retinal Image Quality In Pseudophakic Eyes," Invest Ophth Vis Sci, 52(14), pp. 6184-6184, (2011).

[10] A. G. Marrugo, M. S. Millan, G. Cristóbal, S. Gabarda, and H. C. Abril, "Anisotropy-based robust focus measure for non-mydriatic retinal imaging," J. Biomed. Opt., 17(7), p. 076021, (2012).

[11] M. Subbarao, T. Choi, and A. Nikzad, "Focusing techniques," Optical Engineering, 32(11), pp. 28242836, (1993).

[12] P. Bedggood, M. Daaboul, R. Ashman, G. Smith, and A. Metha, "Characteristics of the human isoplanatic patch and implications for adaptive optics retinal imaging," J. Biomed. Opt., 13(2), p. 024008, (2008).

[13] G. Wallace, “The JPEG still picture compression standard," IEEE Trans on Consumer Electronics, 38(1), pp. xviii-xxxiv, (1992).

[14] J. Ramirez, A. Garcia, P. Fernandez, L. Parrilla, and A. Lloris, "A new architecture to compute the discrete cosine transform using the quadratic residue number system," IEEE International Symposium on Circuits and Systems, 5, pp. 321-324, (2000).

[15] C. Stewart, Chia-Ling Tsai, and B. Roysam, "The dual-bootstrap iterative closest point algorithm with application to retinal image registration," Medical Imaging, IEEE Transactions on, 22(11), pp. 13791394, (2003).

[16] L. Fernández-Vega, J. F. Alfonso, and T. Villacampa, "Clear lens extraction for the correction of high myopia," Ophthalmology, 110(12), pp. 2349-2354, (2003).

[17] M. Niemeijer, J. Staal, B. van Ginneken, M. Loog, and M. D. Abramoff, "Comparative study of retinal vessel segmentation methods on a new publicly available database," presented at the Medical Imaging 2004: Image Processing, 2004, 5370, pp. 648-657 (2004).

[18] A. Hoover and M. Goldbaum, "Locating the optic nerve in a retinal image using the fuzzy convergence of the blood vessels," Medical Imaging, IEEE Transactions on, 22(8), pp. 951-958, (2003).

[19] B. Al-Diri, A. Hunter, D. Steel, M. Habib, T. Hudaib, and S. Berry, "A reference data set for retinal vessel profiles," presented at the Engineering in Medicine and Biology Society, 2008. EMBS 2008. 30th Annual International Conference of the IEEE, pp. 2262-2265 (2008).

\section{Introduction}

Non-mydriatic retinal imaging is nowadays a standard tool for diagnosis and progression assessment of ophthalmic diseases [1]. Patients that have undergone prior cataract surgery may require further eye fundus exploration. In this work we consider the changes produced in the fundus images of patients that have undergone this kind of surgery. Several factors concerning the optical system of the eye, the ocular media, and the implanted intraocular lens (IOL), may have an influence on the quality of the image 
acquired with the retinal camera. We propose a measure to quantitatively assess those changes of image quality.

Retinal image quality has conventionally been related to the quality of the eye fundus image in terms of the best possible acquisition [2], [3]. In essence, a retinal image that is blurry, has poor illumination, has reflection artifacts, among other unwanted effects should be discarded and another acquisition should be considered [4], [5]. However, in this work we are not considering this situation. Most of these unwanted effects can be compensated during acquisition, but it is important to note that the optics of the eye (the cornea and the crystalline lens) is part of the imaging system. Therefore, this critical part of the imaging system cannot be controlled by the retinal camera, and should be considered when acquiring eye fundus images.

Papers on retinal image quality after IOL implantation refer to the impact on the quality of a patient's vision after surgery[6]-[9] rather than to the quality of a digital fundus image of a pseudophakic eye as obtained with a retinal camera for common retinal examination. To the best of our knowledge, this specific issue has not been addressed yet.

We illustrate our pilot study with several cases taken retrospectively from the clinical practice. A pair of eye fundus images represents each case: one taken before and the other after surgery. A quality measure based on a calculation of image anisotropy [10] is applied to each image. In our quantitative analysis, this metric is computed in a few sub-windows -located approximately in the same region of both images, which in several cases have been previously registered for better spatial alignment and comparison-.

The results show that, in general, the retinal images of cataractous eyes are poor quality, dim and affected by blur. After surgery with IOL implant, the retinal image is commonly much brighter, sharp and higher quality. However, several conditions may alter this rule of thumb. The presence of astigmatism, the multifocality of the intraocular implant, among other factors discussed in the paper, may hinder eye fundus image quality. We analyse the suitability of the proposed anisotropy-based measure to address a quantitative description of those image quality changes.

\section{Method}

A measure of focus in non-mydriatic retinal imaging based on a calculation of image anisotropy was proposed in [10] and proved to be a robust, accurate and effective metric to find out the best focusing conditions in which an eye fundus image must be acquired with a non-mydriatic retinal camera. The calculation of image anisotropy was evaluated from the directional variance of the normalized discrete cosine transform (DCT). The results obtained with such anisotropy-based measure were in good agreement with those obtained with former works [11]. In this section we briefly review the principles of the anisotropy-based measure [10] used in this work as a metric to assess the changes in quality occurred in retinal images acquired before and after intraocular lens implantation.

\section{2.a. Anisotropy-based focus measure to assess retinal image quality variations}

Let $f(x, y)$ for $x=0,1,2 \ldots, M-1$ and $y=0,1,2 \ldots, N-1$ denote the $M \times N$ focused image of a planar object and $g_{i}(x, y)$ a set of images with $i=\{1,2\}$ typically corresponding to a pre- and post-operatory retinal image pair, recorded using given camera parameter settings. Although the eye fundus is a curved surface, in our case $f(x, y)$ corresponds to a small region of the fundus, thus considered as an isoplanatic patch [12]. The acquired images can be expressed by convolution

$$
g_{i}(x, y)=\left(f * h_{i}\right)(x, y), \quad i=1,2,
$$

where $h_{i}(x, y)$ is the point spread function (PSF) of the blur in the $i$ th observation, and * is the standard convolution operator. In practice, all $h_{i}(x, y)$ have an unknown low-pass filter effect. Let $g(x, y)$ denote an $M \times N$ image and its discrete cosine transform (DCT), denoted by $G(u, v)=\operatorname{DCT}[g(x, y)]$, given by the equation

$$
G(u, v)=\sum_{y} \sum_{x} g(x, y) \alpha(u) \alpha(v) \cos \left[\frac{(2 x+1) u \pi}{2 M}\right] \cos \left[\frac{(2 y+1) v \pi}{2 N}\right],
$$


where

$$
\alpha(\xi)= \begin{cases}\sqrt{\frac{1}{A}} & , \text { for } \xi=0, \\ \sqrt{\frac{2}{A}} & \text {,otherwise, }\end{cases}
$$

where $A=\{M, N\}$ depending on variables $u, v$, and $\xi$ is a dummy variable for the purpose of simplifying equation (2); i.e. $\alpha(u=0)=\sqrt{1 / M}, \alpha(u=1)=\sqrt{2 / M}$, and so on. Low-order basis functions represent low spatial frequencies, while those of higher orders represent the higher spatial frequencies (Fig. 1). The DCT is closely related to the discrete Fourier transform (DFT), a standard tool in signal processing. However, the DCT has a greater energy compaction property than the DFT, i.e., most of the image information tends to be concentrated in a few low-frequency DCT coefficients. This is also why the JPEG compression standard is based on the DCT [13]. In addition, many efficient schemes for the computation of DCT exist, and hardware implementations are commonly available [14].

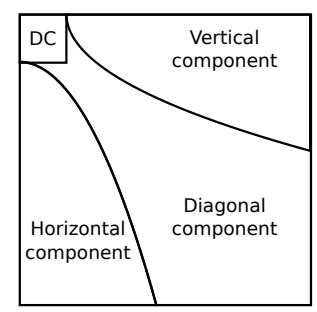

(a)

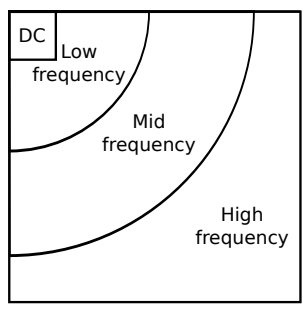

(b)

Fig. 1 Relationship between DCT coefficients and frequency components of an image

The normalized DCT of an image, defined as

$$
\tilde{G}(u, v)=\frac{|G(u, v)|}{\sum_{(u, v)}|G(u, v)|},
$$

allows invariance to changes in the global contrast of the image. Generally, in the spectrum of a focused, sharp image, there are some favoured orientations (anisotropy) in the mid- and low-frequency coefficients. In contrast, in a blurred image, the high- and mid-frequency content of the spectrum is more uniformly distributed. Moreover, in the blurred spectrum the coefficients related to high frequency, frequently affected by noise, usually decrease significantly. The anisotropy-based measure aims to quantify the influence of blur on image anisotropy based on the normalized DCT of the image. Let us consider an $N \times N$ image and $\lambda_{j}$, for $j=1,2,3$, a vector along one of the three main orientations of its spectrum depicted in Fig. 2. We restrict our study to angular partitions of the spectrum roughly equivalent to vertical, diagonal, and horizontal components of the image space. Our measure of anisotropy mainly consists in calculating a difference of weighted coefficients along these orientations.

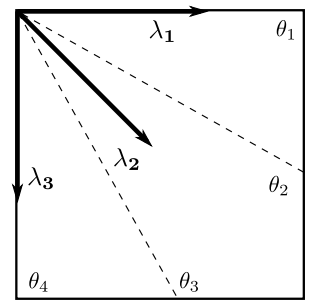

Fig. 2 Vectors along the main directions of the DCT. 
Let $\widetilde{G}=\left\{\tilde{G}(u, v): \theta=\arctan \left(\frac{v}{u}\right), \theta_{j} \leq \theta<\theta_{j+1}, j=1,2,3\right\}$ be the set of DCT coefficients located between $\theta_{j}$ and $\theta_{j+1}$ angles, for $\theta_{j} \in\left\{0^{\circ}, 30^{\circ}, 60^{\circ}, 90^{\circ}\right\}$. The function $\psi_{\lambda_{j}}($.$) takes as input \tilde{G}_{j}$, performs orthogonal projection of all its elements along vector $\lambda_{j}$, and averages the elements that after projection fall on the same discrete $(u, v)$ coordinates. With $\psi_{\lambda_{j}}($.$) the information around each main orientation can be$ compacted in a one-dimensional vector of $N$ elements, which in turn, can be stacked to form the matrix

$$
\Psi=\left[\begin{array}{ccc}
\psi_{\lambda_{1}}^{1} & \psi_{\lambda_{2}}^{1} & \psi_{\lambda_{3}}^{1} \\
\psi_{\lambda_{1}}^{2} & \psi_{\lambda_{2}}^{2} & \psi_{\lambda_{2}}^{2} \\
\vdots & \vdots & \vdots \\
\psi_{\lambda_{1}}^{N} & \psi_{\lambda_{2}}^{N} & \psi_{\lambda_{3}}^{N}
\end{array}\right],
$$

where the first element of each vector does not really convey any directional information of the image because it corresponds to the $d c$ coefficient. However, it is kept in the matrix for the sake of completeness. The measure of anisotropy is obtained from $\boldsymbol{\Psi}$ by calculating the variance of the weighted sum of the columns, computed as the matrix product $\mathbf{w} \Psi$,

$$
S_{a}(g)=\operatorname{Var}(\mathbf{w} \Psi)=\mathrm{E}\left[(\mathbf{w} \Psi-\mu)^{2}\right],
$$

where $\mathbf{w}=w_{1}, w_{2}, \ldots, w_{N}, \mathbf{E}$ is the expected value and $\mu$ is the mean of the matrix product $\mathbf{w} \Psi$. Vector $\mathbf{w}$ can be regarded as a weighting procedure to increase robustness to noise and illumination variation. Following the optimisation procedure described in [10] a suitable vector $\mathbf{w}$ assigns higher weights for mid frequencies than for low and high frequencies, in analogy to a band-pass filter.

\section{2.b. Implementation}

To assess the image quality throughout the field of view, we manually select 6 regions or analysis windows of $128 \times 128$ pixels within the retinal image. The windows are distributed counterclockwise for the right eye fundus images, starting from the optic nerve head, window $\# 1$, and continuing throughout the blood vessel vasculature (Figs. 3, 5, 6, 7). For left eye fundus images, the windows are distributed clockwise (Fig. 4, 8). There are no constraints for partial overlapping of the $128 \times 128$ windows. For speed, the anisotropy measure is carried out by dividing the $128 \times 128$ window into subwindows of $16 \times 16$ pixels. The measure is computed in the following manner:

1. Every $128 \times 128$ window is divided into non-overlapping sub-windows of size $16 \times 16$. This size is chosen so that the most basic structures of the image fit in the subwindows.

2. Each subwindow image is transformed with the normalized DCT, and the measure $S_{a}$ is computed.

3. An overall $\tilde{S}_{a}$ is computed by taking the mean of all $S_{a}$ values from the subwindows.

The resulting measure for each region is saved as a vector of 6 elements and normalized so that the maximum value is 1 . The purpose of normalization is for comparing the resulting measure from two or more images.

\section{Results using real pre- and post-operative eye fundus image pairs}

In this section, we present the results obtained for six cases (figs. 3 to 8) chosen to illustrate some situations that may appear in the clinical practice when a patient is explored with a non-mydriatic fundus camera before and after undergoing cataract surgery with IOL implant. Two cameras have been used: Ultra-widefield (UWF ${ }^{\mathrm{TM}}$ ) Optos California (figs. 3,7,8) and Zeiss VISUCAM ${ }^{\circledR} 500$ (figs. 4,5,6). A variety of IOLs ranging from monofocal, toric, bifocal, and trifocal, have been included. Each case consists of a pair of images, taken before and after surgery. When necessary, the images were registered using the algorithm described in reference [15] to make them spatially aligned before applying the DCT (figures 3,7,8). The anisotropy-based measurement was computed in six manually selected windows, mainly placed on the optical disc and along the blood vessels. This selection of windows takes into account that the possible variations in the anisotropy would be more prominent in these areas of the image than in other areas without any visible structure. 
The results of all the six cases are presented through figures 3 to 8 with the same distribution of information: pre-operative image, post-operative image, available data, and a bar diagram with the distribution of the anisotropy-based measurement in the $\{1, . ., 6\}$ selected windows of the pre- and postoperative images. The black bars correspond to pre-operative images, whereas the grey bars to postoperative.

Although the cases shown in figures 3 to 8 do not correspond to a prospective study, the available data allow us to to illustrate the potential of the anisotropy-based measure to quantify the changes in fundus image quality that can appear before crystalline lens removal and after intraocular lens implantation.

Firstly, let us describe the cases. Figure 3 corresponds to a case of clear lens extraction in a 47-year patient and its replacement with a bifocal IOL (with $+3 \mathrm{D}$ add power) for far and near distinct vision. The use of this type of surgery as a refractive modality in patients without cataracts and reduced accommodation capability (typically at their late forties and older) has increased because it presents several advantages in comparison with corneal refractive surgery, particularly in patients with high degrees of myopia [16] hyperopia and astigmatism. An implant with multifocal or accommodating IOLs may compensate for original pre-operative refractive errors, alleviate presbyopia, and overcome the future need for cataract surgery. Looking at the pre- and post- operative images of Fig. 3, it is hard to say which one is better quality.

The rest of figures ( 4 to 8 ) correspond, in their pre-operative status, to a variety of cataractous eyes. Figure 4 corresponds to a patient that suffered from astigmatism in addition to cataract. A toric IOL was implanted to the patient for astigmatism compensation. The background illumination is severely nonuniform in the eye fundus images of fig. 4, particularly in the post-operative image. Despite this fact, a watchful eye would appreciate more detail and less blur in the post- than in the pre-operative image. Figures 5 to 8 correspond to patients that were implanted with different types of IOLs for distinct intermediate/far vision (bifocal IOL with add power $1.75 \mathrm{D}$ in fig. 5), near/intermediate/far vision (trifocal IOL with add powers 3.25 and 2.17D in fig. 6) and just far vision (monofocal IOLs in fig. 7 and 8). The images of the latter two cases (figs. 7 an 8) were taken with the same camera to patients with cataract of different severity. After surgery, a more significant change occurred in the fundus image of the patient of fig. 7.

Secondly, let us discuss on the results obtained from calculation of the anisotropy-based measure, starting from the results with immediate interpretation. In figs. 5 to 7, for which the quality of the pre-operative images is low and appears extraordinarily improved in the post-operative image, much higher values of the anisotropy-based measure are obtained in all the six selected windows of the post-operative images in comparison with those of the pre-operative image. In contrast with this, slight differences between the pre- and post-operative image quality can be also appreciated by the anisotropy-based measure. This is the case of fig. 8, with pre- and post-operative image quality changes in all six windows clearly shorter than in figs 5-7. And yet, the anisotropy-based measure is sensitive to aberration compensation (astigmatism with a toric implant in fig. 4) and so, to the details emerged in most of the windows (\#1, 4, 5, 6) of the post-operative image despite its markedly non-uniform background.

The case shown in fig. 3 deserves a more detailed comment. This case is the only example where the quality of the post-operative image is not better than the pre-operative image. And this result is obtained consistently in all the six selected windows, for which the anisotropy-based measure in the pre-operative image is somewhat higher than in the post-operative image. The pre-operative image is of relatively good quality, and not dim or blurred, in accordance to the presence of a clear crystalline lens and unlike the rest of pre-operative cases. After surgery, the imaging characteristics of a bifocal IOL give rise to the formation of two fundus images simultaneously. Although the retinal camera acquires the image formed by the distance power (used for far vision), the other image (formed by the near power, used for near vision) affects the acquired eye fundus image with a loss of contrast. This could be a reason for not achieving better quality in the post-operative image of case shown in fig. 3. 

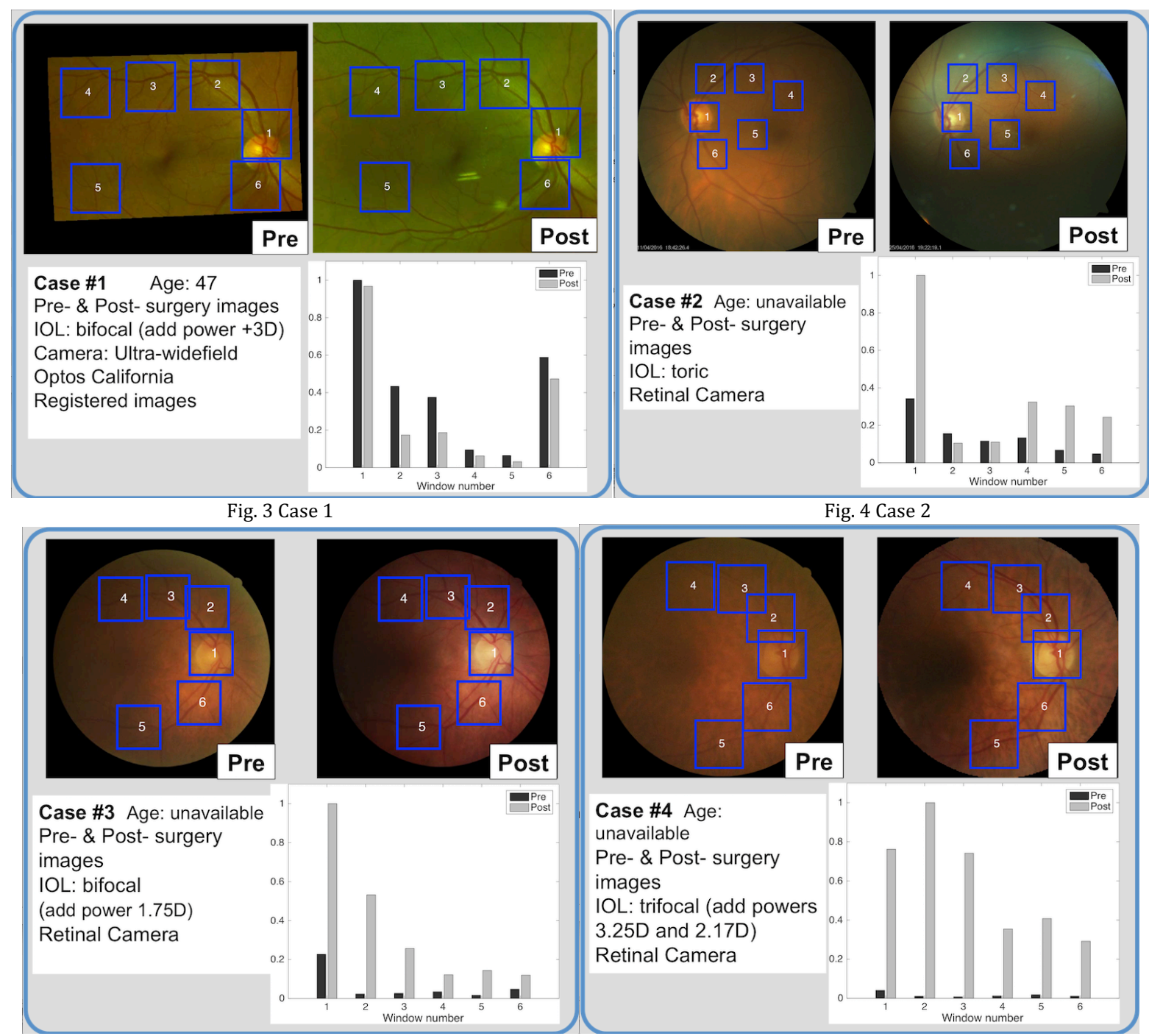

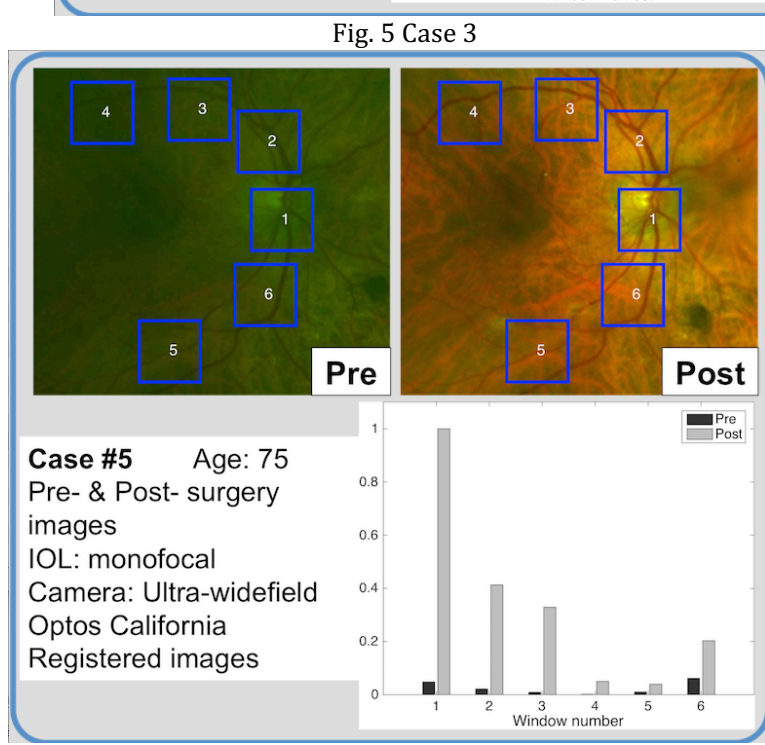

Fig. 7 Case 5

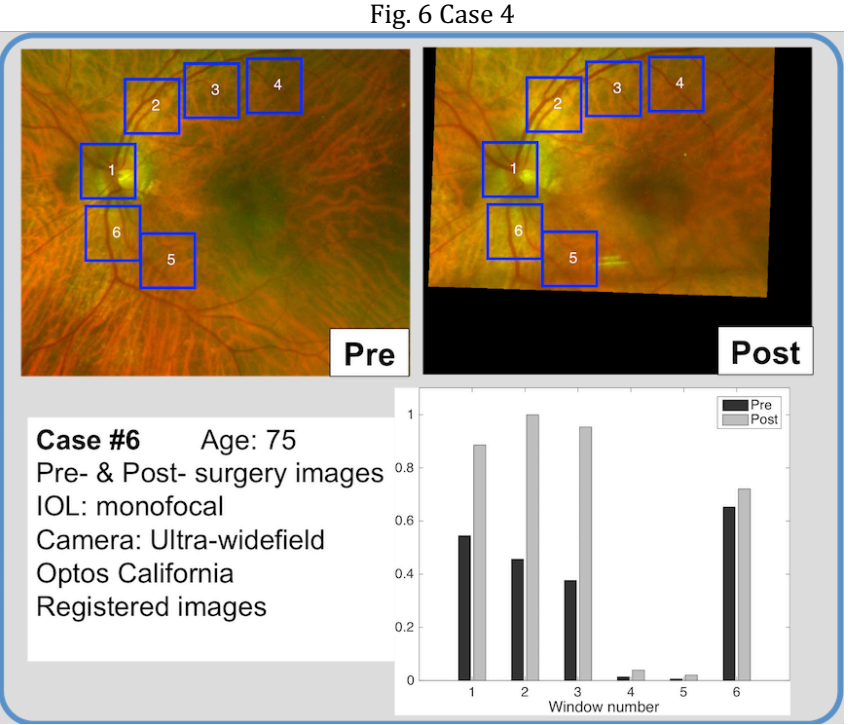

Fig. 8 Case 6 


\section{Conclusions}

In general, the retinal images of cataractous eyes are poor quality, dim and affected by blur. After surgery with intraocular lens implant, the retinal image is usually much brighter, sharp and higher quality. However, several conditions may alter this rule of thumb. The presence of astigmatism, or the multifocal design of the intraocular implant, among other factors, may hinder post-operative eye fundus image quality. An anisotropy-based measure, as proposed in this work, has revealed to be a suitable tool to quantitatively assess such image quality changes between pre-and post-operative fundus images.

Although this work does not present an exhaustive study, it brings into consideration the influence of IOL characteristics on the eye fundus image quality. This issue can also appear in eyes that have not undergone cataract but corneal refractive surgery, or both. To the best of our knowledge, these kind of changes have not been sufficiently studied and have not introduced yet in databases -such as current DRIVE [17], STARE [18] and REVIEW [19]- widely used in image analysis for construction and testing of vessel detection algorithms. We consider it deserves further study in varied clinical conditions.

\section{Acknowledgements}

The authors thank Institut d'Oftalmologia Clínica de Girona and Vallès Ophthalmology Research for providing original images and David Amorós for his collaboration. One of the authors (F. A.-B.) was an employee of Vallès Ophthalmology Research - Institute of Medical and Surgical Ophthalmology at the time of the research work reported in this paper. The authors acknowledge the financial support of Spanish MINECO and FEDER funds under project ref. DPI2016-76019-R, and Centre de Cooperació i Desenvolupament at UPC under project ref. CCD2018-U005, and Universidad Tecnológica de Bolívar (Colombia). Parts of this work were presented at the IX Iberoamerican Optics Meeting (Abstract Book of RIAO-OPTILAS 2016, Moraga, P. and Saavedra, C.; 21-25 November; CEFOP-UdeC-Concepción - Chile, 2016, p. 237). 